\title{
Analysis of a Nonlinear High-Gain Observer in the Presence of Measurement Noise
}

\author{
Alexis A. Ball and Hassan K. Khalil \\ Department of Electrical and Computer Engineering \\ Michigan State University \\ East Lansing, Michigan 48824-1226 \\ Email: \{ballalex, khalil\}@egr.msu.edu
}

\begin{abstract}
Motivated by the sensitivity of high-gain observers to measurement noise, this paper presents a high-gain observer with a nonlinear gain that takes the form of a piecewise linear function. The piecewise function is designed to have three linear regions, with the two outermost sectors chosen to elicit the desired behavior in the transient and steadystate responses, respectively. In order to overcome the tradeoff between fast state reconstruction and measurement noise attenuation, a larger observer gain is generated during the transient response than in the steady-state response. Thus, by reducing the observer gain after achieving satisfactory state estimates, the effect of noise on the steady-state performance is reduced. Moreover, the observer presented in this paper is shown to surpass the system performance achieved when using comparable observers. The proof argues boundedness and ultimate boundedness of the closed-loop system under the proposed output feedback.
\end{abstract}

\section{INTRODUCTION}

High-gain observers have developed into an important topic in state estimation and output feedback control of nonlinear systems, beginning with papers such as [1] and [2]. In the absence of measurement noise, this technique robustly estimates the derivatives of the output while achieving fast convergence [1]. Moreover, for a sufficiently high observer gain and a globally bounded controller, the high-gain observer is able to recover the system performance achieved with the state feedback control. Refer to [3] for a survey on high-gain observers.

However, observer theory reveals that a trade-off exists between the measurement noise sensitivity and the speed of state reconstruction [4]. As the observer gain is increased, the bandwidth of the observer is extended. As the bandwidth increases the high-gain observer asymptotically approaches the behavior of a differentiator, exacerbating the presence of measurement noise. The authors of [5], in the context of discrete-time models, exploited this knowledge by designing a switched filter composed of two linear filters (one for the transient response and the other for the steady-state response); the value of the estimation error determines which filter is active. The idea is to use a large filter gain (increasing the filter bandwidth) during the transient behavior to elicit a fast recovery of the state estimates. The filter with the smaller

This work was supported in part by the National Science Foundation under grant number ECCS-0725165. gain is active once the estimation error has reached a steadystate threshold, reducing the filter bandwidth and preventing a large magnification of the measurement noise. In [6] the authors seek to minimize the effect quantization error has on shaft encoder measurements by introducing a dead-zone nonlinearity into the state estimation scheme. The dead-zone nonlinearity is used to alternate "smoothly" between varying filter bandwidths to initially achieve fast state estimation, and ultimately minimizing the quantization error.

Recently, Khalil and colleagues have studied the effect of measurement noise in [7]-[9]. In particular, there exists a trade-off between reducing the estimation error in steadystate, and achieving fast state reconstruction [8]. It is shown that the steady-state estimation error has a component due to modeling uncertainty, which can be attenuated by increasing the gain. Furthermore, the error has a component due to measurement noise that is amplified by increasing the gain. This trade-off constrains the observer gain, which reduces the observer's ability to quickly reconstruct the states. Both [8] and [9] sought to construct observers to eliminate the manifestations of the trade-off in the system states. For instance, a switched-gain observer is proposed in [8] to force a large gain during the transient period for fast state reconstruction, and allow for a smaller gain once the states are satisfactorily estimated to reduce the effect of noise on the steady-state performance. However, a number of complications are generally associated with a switched system. The time in which the gains are switched, trigger threshold, and system peaking are all issues that must be addressed. Both from an analysis and design/implementation prospective, using a switched observer can be tedious.

Inspired by the work in [6] and [8], the authors of [9] attempt to mitigate the above trade-off with the careful construction of a high-gain observer with a nonlinear gain. The nonlinearity is chosen to have a higher observer gain during the transient period and a lower gain afterwards, thus overcoming the trade-off between fast state reconstruction and measurement noise attenuation. The high-gain observer in [9] is capable of producing similar results to the switched observer, however, the transient response for the nonlinear-gain observer is slower. Nonetheless, a nonlinear gain approach has the potential to achieve better system performance than previously demonstrated schemes, while 
bypassing the complications associated with switching. The results provided in this paper will show that the newly introduced observer with the piecewise linear gain is able to achieve enhanced system performance over the observer presented in [9].

The purpose of this work is to construct a new high-gain observer containing a nonlinear gain that takes the form of a piecewise linear function with three distinct linear regions. The innermost section of the piecewise function is designed to accelerate the transition between the desired transient and steady-state observer gains. The outermost sections are chosen to correspond to the desired transient and steadystate responses, respectively. By constructing the observer gain in this manner, we can achieve fast state estimation and reduced steady-state error. Furthermore, the observer is devised such that the behavior of the innovation process can be controlled separately from the other system states. This is accomplished by assigning one fast eigenvalue, with the remaining eigenvalues chosen relatively slow. Without this key step, the stability analysis for the proposed observer is unattainable. The bulk of this paper is dedicated to the analysis of the proposed high-gain observer and the proof for the closed-loop system dynamics. The discussion concludes with a simulation comparing the system performance under the linear and two nonlinear-gain high-gain observer designs.

\section{Problem Formulation And System Description}

As in [9], consider the nonlinear system

$$
\begin{aligned}
\dot{z} & =\psi(x, z, \varsigma, u) \\
\dot{x} & =A x+B \phi(x, z, \varsigma, u) \\
y & =C x+v \\
w & =\Theta(x, z, \varsigma)
\end{aligned}
$$

where $z \in \mathbb{R}^{l}$ and $x \in \mathbb{R}^{n}$ are the system states, $y \in \mathbb{R}$ and $w \in \mathbb{R}^{s}$ are the measured outputs, $u \in \mathbb{R}$ is the control input, $\varsigma(t) \in \mathbb{R}^{p}$ represents the exogenous signals, and $v(t) \in \mathbb{R}$ is the measurement noise. The function $\phi(x, z, \varsigma, u)$ may not be known. The triple $(A, B, C)$ represents a chain of $n$ integrators, where it is assumed that $n \geq 2$. Possible sources for the model (1) - (4) include mechanical systems, electromechanical systems, and systems that can be placed in the normal form satisfying the conditions of input-output linearizability.

\section{Assumption 1:}

- $\dot{\varsigma}(t)$ and $v(t)$ are measurable functions of $t$ and bounded, where $|v(t)| \leq \mu$

- $\phi, \psi$, and $\Theta$ are locally Lipschitz in their arguments, uniformly in $\varsigma$

The state feedback controller takes the following form

$$
\begin{aligned}
\dot{\theta} & =\Gamma(\theta, x, w, \varsigma) \\
u & =\gamma(\theta, x, w, \varsigma)
\end{aligned}
$$

and meets the requirements listed in Assumption 2.

\section{Assumption 2:}

- $\Gamma$ and $\gamma$ are locally Lipschitz functions in their arguments, uniformly in $\varsigma$, over the domain of interest

- $\Gamma$ and $\gamma$ are globally bounded functions of $x$

Let the closed-loop system (1) - (4) under the state feedback controller (5) - (6) be denoted as

$$
\dot{\chi}=f_{r}(\chi, \varsigma)
$$

where

$$
\chi=\left[\begin{array}{c}
x \\
z \\
\theta
\end{array}\right] \in \mathbb{R}^{N} \text { and } f_{r}(\chi, \varsigma)=\left[\begin{array}{c}
A x+B \phi(x, z, \varsigma, \gamma) \\
\psi(x, z, \varsigma, \gamma) \\
\Gamma(\theta, x, w, \varsigma)
\end{array}\right]
$$

\section{Assumption 3:}

- The closed-loop system (7) is globally uniformly asymptotically stable with respect to a compact positively invariant set $\mathcal{A}$, uniformly in $\varsigma$

- $\phi(x, z, \varsigma, \gamma)$ is zero in $\mathcal{A}$, uniformly in $\varsigma$

In extending the definition of stability to a compact positively invariant set instead of just an equilibrium point, a wider variety of problem formulations can be encapsulated in the above setup.

The high-gain observer is defined as

$$
\dot{\hat{x}}=A \hat{x}+B \phi_{0}(\hat{x}, w, \varsigma, u)+h\left(y-\hat{x}_{1}\right)
$$

where

$$
\begin{aligned}
h_{i}\left(y-\hat{x}_{1}\right)=\alpha_{i} & {\left[g_{1}^{i}\left(y-\hat{x}_{1}\right)+d_{1}\left(g_{2}^{i}-g_{c i}\right) \mathrm{sat}\left(\frac{y-\hat{x}_{1}}{d_{1}}\right)\right.} \\
& \left.+d_{2}\left(g_{c i}-g_{1}^{i}\right) \mathrm{sat}\left(\frac{y-\hat{x}_{1}}{d_{2}}\right)\right]
\end{aligned}
$$

$d_{2}>d_{1}>0$, and "sat" denotes the saturation function defined as

$$
\operatorname{sat}(e)= \begin{cases}e, & \text { if }|e| \leq 1 \\ \operatorname{sign}(e), & \text { if }|e|>1\end{cases}
$$

The function $\phi_{0}$ is a nominal model of $\phi$. The observer gains are defined as

$$
g_{1}=1 / \varepsilon_{1} \text { and } g_{2}=1 / \varepsilon_{2}, \text { where } \varepsilon_{1}<\varepsilon_{2}
$$

and chosen to correspond to the desired transient and steadystate responses, respectively. Both $\varepsilon_{1}$ and $\varepsilon_{2}$ are small positive parameters. The parameter $d_{1}$ is defined such that the observer gain is the smaller value $g_{2}$ for $\left|x_{1}-\hat{x}_{1}\right| \leq d_{1}$. The expression for $g_{c}$ is given as

$$
g_{c i}=\frac{d_{2} g_{1}^{i}-d_{1} g_{2}^{i}}{d_{2}-d_{1}}
$$

The nonlinear gain presented in [9] is a special case that can be obtained by setting $d_{2}=d_{1}$ in (8). A plot of the nonlinear gain function is shown in Fig. 1. The $\alpha_{i}$ 's are designed such that the roots of

$$
s^{n}+\alpha_{1} s^{n-1}+\cdots+\alpha_{n-1} s+\alpha_{n}=0
$$

are real and negative, with one fast root and $(n-1)$ slow real roots. In this case, (9) is written as

$$
\left(s^{n-1}+\beta_{1} s^{n-2}+\cdots+\beta_{n-2} s+\beta_{n-1}\right)(s+\lambda)=0
$$


where the first polynomial is Hurwitz with $\mathcal{O}(1)$ real roots and $\lambda \gg 1$. Relating (9) to (10), it can be seen that $\alpha_{1}=\lambda+\beta_{1}, \alpha_{i}=\beta_{i-1} \lambda+\beta_{i} \forall 1<i<n$, and $\alpha_{n}=\beta_{n-1} \lambda$.

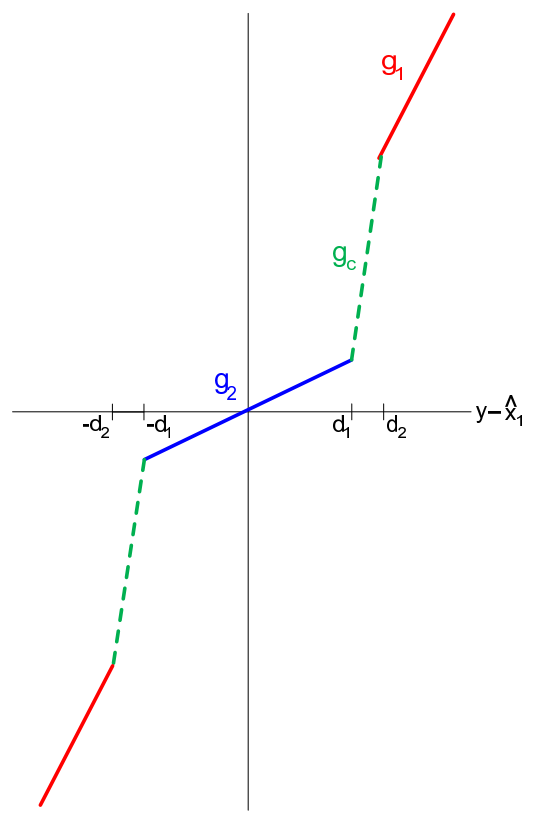

Fig. 1. Plot of the nonlinear gain function

\section{Assumption 4:}

- $\phi_{0}$ is locally Lipschitz in its arguments, uniformly in $\varsigma$, over the domain of interest

- $\phi_{0}$ is globally bounded in $x$ and zero in $\mathcal{A}$

The output feedback controller is obtained by replacing $x$ in (5) - (6) with $\hat{x}$.

\section{OBSERVER DYNAMICS}

For the closed-loop system analysis, the observer dynamics are replaced by the equivalent dynamics of the scaled estimation error:

$$
\eta=D\left(\varepsilon_{1}\right)[x-\hat{x}]
$$

where $D\left(\varepsilon_{1}\right)=\operatorname{diag}\left[1, \varepsilon_{1}, \cdots, \varepsilon_{1}^{n-1}\right]$. The closed-loop system under the output feedback controller can be written as

$$
\begin{gathered}
\dot{\chi}=f\left(\chi, \varsigma, D^{-1}\left(\varepsilon_{1}\right) \eta\right) \\
=\left[\begin{array}{c}
A x+B \phi\left(x, z, \varsigma, \gamma\left(\theta, x-D^{-1}\left(\varepsilon_{1}\right) \eta, w, \varepsilon\right)\right) \\
\psi\left(x, z, \varsigma, \gamma\left(\theta, x-D^{-1}\left(\varepsilon_{1}\right) \eta, w, \varepsilon\right)\right) \\
\Gamma\left(\theta, x-D^{-1}\left(\varepsilon_{1}\right) \eta, w, \varepsilon\right)
\end{array}\right] \\
\varepsilon_{1} \dot{\eta}=A_{0} \eta+B_{0} v+\varepsilon_{1}^{n} B \delta\left(\chi, \varsigma, w, D^{-1}\left(\varepsilon_{1}\right) \eta\right) \\
+\bar{h} \delta_{1}\left(\eta_{1}+v\right)
\end{gathered}
$$

where

$$
\begin{gathered}
A_{0}=\left[\begin{array}{ccccc}
-\alpha_{1} & 1 & \cdots & \cdots & 0 \\
-\alpha_{2} & 0 & 1 & \cdots & 0 \\
\vdots & & & \ddots & \vdots \\
-\alpha_{n-1} & \cdots & \cdots & 0 & 1 \\
-\alpha_{n} & \cdots & \cdots & \cdots & 0
\end{array}\right], B_{0}=\left[\begin{array}{c}
-\alpha_{1} \\
-\alpha_{2} \\
\vdots \\
-\alpha_{n-1} \\
-\alpha_{n}
\end{array}\right] \\
\bar{h}_{i}=\alpha_{i}\left[1-\left(\frac{\varepsilon_{1}}{\varepsilon_{2}}\right)^{i}\right] \text { and } \\
\delta_{1}\left(\eta_{1}+v\right)=\frac{d_{1} d_{2}}{d_{2}-d_{1}}\left(\operatorname{sat}\left(\frac{\eta_{1}+v}{d_{1}}\right)-\operatorname{sat}\left(\frac{\eta_{1}+v}{d_{2}}\right)\right)
\end{gathered}
$$

Note that $\left|\delta_{1}\right| \leq d_{1}$. The function $\delta\left(\chi, \varsigma, w, D^{-1}\left(\varepsilon_{1}\right) \eta\right)$ is defined as $\phi(x, z, \varsigma, \gamma(\theta, \hat{x}, w, \varsigma))-\phi_{0}(\hat{x}, w, \varsigma, \gamma(\theta, \hat{x}, w, \varsigma))$, and the matrix $A_{0}$ is Hurwitz. The equations (12) - (13) resemble a model appearing in the standard singularly perturbed form, as shown in [10]. The primary difference between this system and the standard form is the presence of the negative powers of $\varepsilon_{1}$ in the term $D^{-1}\left(\varepsilon_{1}\right) \eta$. However, $\delta$ is a globally bounded function in $\hat{x}$, implying that it is also globally bounded in $D^{-1}\left(\varepsilon_{1}\right) \eta$. This property allows us to extend the analysis associated with standard singularly perturbed systems to the case involving (12) - (13). The slow dynamics of (12) can be approximated by $\varepsilon_{1}=0$, which yields $\eta=0$. This reduces (12) to the closed-loop system (7) under the state feedback controller (5) - (6). Moreover, the system is globally uniformly asymptotically stable with respect to the compact positively invariant set $\mathcal{A}$. Then, according to a converse Lyapunov theorem in [11], there exists a smooth Lyapunov function $V(\chi)$ and three positive definite (with respect to $\mathcal{A}$ ) functions $U_{1}, U_{2}$, and $U_{3}$ all defined in $\mathbb{R}^{N}$ such that

$$
\begin{array}{r}
V(\chi)=0 \Leftrightarrow \chi \in \mathcal{A} \\
U_{1}(\chi) \leq V(\chi) \leq U_{2}(\chi) \\
\lim _{\chi \rightarrow \infty} U_{1}(\chi)=\infty \\
\frac{\partial V}{\partial \chi} f(\chi, \varsigma, 0) \leq-U_{3}(\chi)
\end{array}
$$

Theorem 1: Let Assumptions 1 through 4 hold and consider the closed-loop system (7) with the observer (8). Moreover, let $\mathcal{M}$ be any compact set in $\mathbb{R}^{N}$ and $\mathcal{N}$ be any compact subset of $\mathbb{R}^{n}$, where $\chi\left(t_{0}\right) \in \mathcal{M}$ and $\hat{x}\left(t_{0}\right) \in \mathcal{N}$. Then:

- Given the positive constants $d_{1}, d_{2}>d_{1}$, and $\varepsilon_{f}=$ $\varepsilon_{1} / \varepsilon_{2}<1$, there exist positive constants $\mu^{*}<d_{1}, \lambda^{*}$, and $c_{a}$ such that for $\mu<\mu^{*}$ there is a constant $\varepsilon_{a}=$ $\varepsilon_{a}(\mu)>c_{a} \mu^{1 / n}$ with $\lim _{\mu \rightarrow 0} \varepsilon_{a}(\mu)=\varepsilon_{2}^{*}>0$, such that for each $\varepsilon_{2} \in\left(c_{a} \mu^{1 / n}, \varepsilon_{a}\right]$ the trajectories of the closed-loop system are bounded for all $t \geq 0$.

- There exists $\mu_{1}^{*}>0$ and a class $\mathcal{K}$ function $\rho_{1}$ such that for every $\mu<\mu_{1}^{*}$ and every $\Upsilon_{1}>\rho_{1}(\mu)$, there are constants $T_{\Upsilon}=T_{\Upsilon}\left(\Upsilon_{1}\right) \geq 0$ and $\varepsilon_{b}=\varepsilon_{b}\left(\mu, \Upsilon_{1}\right)>$ $c_{a} \mu^{\frac{1}{n}}$, with $\lim _{\mu \rightarrow 0} \varepsilon_{b}\left(\mu, \Upsilon_{1}\right)=\varepsilon_{b}^{*}\left(\Upsilon_{1}\right)>0$, such that for each $\varepsilon_{2} \in\left(c_{a} \mu^{\frac{1}{n}}, \varepsilon_{b}\right]$

$$
\max \left\{|\chi(t)|_{\mathcal{A}},\|x(t)-\hat{x}(t)\|\right\} \leq \Upsilon_{1}, \forall t \geq T_{\Upsilon}
$$


- There exist $\mu_{2}^{*}>0$ and a class $\mathcal{K}$ function $\rho_{2}$ such that for every $\mu<\mu_{2}^{*}$ and every $\Upsilon_{2}>\rho_{2}(\mu)$, there is a constant $\varepsilon_{c}=\varepsilon_{c}\left(\mu, \Upsilon_{2}\right)>c_{a} \mu^{\frac{1}{n}}$, with $\lim _{\mu \rightarrow 0} \varepsilon_{c}\left(\mu, \Upsilon_{2}\right)=\varepsilon_{c}^{*}\left(\Upsilon_{2}\right)>0$, such that for each $\varepsilon_{2} \in\left(c_{a} \mu^{\frac{1}{n}}, \varepsilon_{c}\right]$

$$
\left\|\chi(t)-\chi_{r}(t)\right\| \leq \Upsilon_{2}, \forall t \geq t_{0}
$$

where $\chi_{r}(t)$ is the solution of (7) with $\chi_{r}\left(t_{0}\right)=\chi\left(t_{0}\right)$. The last two bullet items are similar to Theorem 1 of [8].

Proof: Due to space limitations, we present only the analysis of the estimation error. The complete proof will be published elsewhere. In order to place the set $\mathcal{M}$ in the interior of $\Omega_{c}=\{V(\chi) \leq c\} \subset \mathbb{R}^{N}$, choose $c>\max _{\chi \in \mathcal{M}} V(\chi)$. The set $\Omega_{c}$ is compact for any choice of $c$. Therefore, the set $\Omega_{c}$ is bounded. We have already established that $\delta$ is a globally bounded function in $D^{-1}\left(\varepsilon_{1}\right) \eta$. Therefore, there is a constant $L_{\delta}>0$ independent of $\varepsilon_{1}$, such that $\|\delta\| \leq L_{\delta}$ for all $\chi \in \Omega_{c}$ and $\eta \in \mathbb{R}^{n}$.

Consider the representation of the fast equation (13) for $\chi \in \Omega_{c}$. To transform the estimation error (13) into the singularly perturbed form, $A_{0}$ and $B_{0}$ are represented as $A_{0}=A_{01} \lambda+A_{02}$ and $B_{0}=B_{01} \lambda+B_{02}$. The procedure from [10] is used to transform the system (13) with the change of coordinates

$$
\left[\begin{array}{c}
\zeta \\
\eta_{1}
\end{array}\right]=T \eta
$$

where

$$
T=\left[\begin{array}{l}
Y \\
Z
\end{array}\right]=\left[\begin{array}{ccccc}
-\beta_{1} & 1 & 0 & \ldots & 0 \\
-\beta_{2} & 0 & 1 & \ldots & 0 \\
\vdots & & & \ddots & \vdots \\
-\beta_{n-1} & 0 & \ldots & \ldots & 1 \\
1 & 0 & \ldots & \ldots & 0
\end{array}\right]
$$

and $T^{-1}=\left[\begin{array}{ll}M & N\end{array}\right]$ with $M \in \mathbb{R}^{n \times(n-1)}$ and $Y \in$ $\mathbb{R}^{(n-1) \times n}$. Applying the change of coordinates to (13) yields

$$
\begin{gathered}
\varepsilon_{1} \dot{\zeta}=Y A_{02} M \zeta+Y B_{02} v+\varepsilon_{1}^{n} Y B \delta+Y \bar{h} \delta_{1} \\
\varepsilon_{1} \dot{\eta}_{1}=-\lambda \eta_{1}+\zeta_{1}-\left(\lambda+\beta_{1}\right) v+\left(\lambda+\beta_{1}\right)\left(1-\frac{\varepsilon_{1}}{\varepsilon_{2}}\right) \delta_{1}
\end{gathered}
$$

where $Y A_{01}=0, A_{02} N=0, Y B_{01}=0, Z A_{01}=0$, $Z A_{01} N=-1, Z A_{02} M=Z, A_{02} N=0$, and $Y A_{02} M$ is by design a Hurwitz matrix. The solution matrix $P$ to the Lyapunov equation $P Y A_{02} M+\left(Y A_{02} M\right)^{T} P=-I$ is symmetric and positive definite. Let the Lyapunov function candidate for (21) be chosen as $W_{1}=\zeta^{T} P \zeta$. It can be shown that

$$
\dot{W}_{1} \leq-\frac{1}{2 \varepsilon_{1}\|P\|} W_{1}, \forall W_{1} \geq L_{W}
$$

for a positive constant $L_{W}$. Thus, the $\zeta$ states are bounded. Using Lemma 1 of [7], it can be shown that $\left|\zeta_{1}\right|$ is ultimately bounded by

$$
c_{0}+k_{1} \mu+k_{2} \varepsilon_{1}^{n} L_{\delta}+k_{3} \frac{\varepsilon_{1}}{\varepsilon_{2}} \lambda d_{1}+k_{4} d_{1}
$$

where $c_{0}$, a constant due to initial conditions, can be made arbitrarily small, and $k_{3}=1-\left(\frac{\varepsilon_{1}}{\varepsilon_{2}}\right)^{n-1}$. The ultimate bound (24) is reached within the interval $\left[t_{0}, t_{0}+T_{1}\left(\varepsilon_{1}\right)\right]$, where

$$
T_{1}\left(\varepsilon_{1}\right) \rightarrow 0 \text { as } \varepsilon_{1} \rightarrow 0
$$

Meanwhile, the next step is to show that the trajectories of (22) reach a positively invariant strip defined as

$$
\left\{\left|x_{1}-\hat{x}_{1}\right| \leq L\right\}
$$

where $0<L<d_{1}$. Using the Lyapunov function $W_{2}=\frac{1}{2} \eta_{1}^{2}$, it can be shown that

$$
\begin{aligned}
\dot{W}_{2}= & -\frac{\lambda}{\varepsilon_{2}} \eta_{1}\left(\eta_{1}+v\right) \\
& -\lambda\left(\frac{1}{\varepsilon_{1}}-\frac{1}{\varepsilon_{2}}\right) \eta_{1}\left(\eta_{1}+v-\delta_{1}\right) \\
& +\eta_{1}\left(\beta_{1}\left(\frac{1}{\varepsilon_{1}}-\frac{1}{\varepsilon_{2}}\right) \delta_{1}-\frac{1}{\varepsilon_{1}} \beta_{1} v+\frac{1}{\varepsilon_{1}} \zeta_{1}\right)
\end{aligned}
$$

It follows from $d_{1}>|v|$ that $\operatorname{sign}\left(\eta_{1}\right)=\operatorname{sign}\left(\eta_{1}+v\right)$ whenever $\left|\eta_{1}+v\right| \geq d_{1}$ and

$$
-\lambda\left(\frac{1}{\varepsilon_{1}}-\frac{1}{\varepsilon_{2}}\right) \eta_{1}\left(\eta_{1}+v-\delta_{1}\right) \leq 0
$$

Using the ultimate bound in (24), after $t_{0}+T_{1}\left(\varepsilon_{1}\right)$,

$$
\dot{W}_{2} \leq-\frac{(1-\theta) \lambda}{\varepsilon_{2}}\left|\eta_{1}\right|^{2}, \forall\left|\eta_{1}\right| \geq U
$$

for

$$
U=\frac{\mu}{\theta}+\frac{\varepsilon_{2}}{\varepsilon_{1}}\left(\frac{c_{1} \mu+c_{2} d_{1}+c_{0}+k_{2} \varepsilon_{1}^{n} L_{\delta}}{\lambda \theta}\right)+\frac{k_{3} d_{1}}{\theta}
$$

where $c_{1}=\beta_{1}+k_{1}, c_{2}=\beta_{1}\left(1-\frac{\varepsilon_{1}}{\varepsilon_{2}}\right)+k_{4}$, and $\theta \in(0,1)$ will be chosen.

To ensure that (26) implies

$$
\left|x_{1}-\hat{x}_{1}+v\right| \leq d_{1}
$$

$d_{1}$ is chosen as $d_{1}>L+\mu$. In order for the strip (26) to be positively invariant, we require the choice of $L$ to be greater than $U$. This requirement leads to the inequality $U<L<$ $d_{1}-\mu$, which can be ensured for all $\mu \in\left(0, \mu^{*}\right], \lambda \geq \lambda^{*}$, and $\varepsilon_{1} \leq 1$ by taking $\theta=1-k \varepsilon_{f}^{n-1}$, where $k \in(0,1)$ and $\varepsilon_{f}=\varepsilon_{1} / \varepsilon_{2}$,

$$
\mu^{*}<\frac{(1-k) \varepsilon_{f}^{n-1} d_{1}}{2-k \varepsilon_{f}^{n-1}}
$$

and

$$
\lambda^{*}>\frac{c_{1} \mu^{*}+c_{2} d_{1}+k_{2} L_{\delta}}{\varepsilon_{f}^{n}\left[(1-k) d_{1}+k \mu^{*}\right]-2 \varepsilon_{f} \mu^{*}}
$$


At other points in the proof, additional restrictions are imposed on $\mu ; \mu^{*}$ is taken as the minimum of all such restrictions.

We will now show that all trajectories reach the strip (26) in finite time. The following inequality originates from (28)

$$
\dot{W}_{2} \leq-\lambda \frac{2 k \varepsilon_{f}^{n}}{\varepsilon_{1}} W_{2}, \forall W_{2} \geq \frac{1}{2} L^{2}
$$

Therefore, the set

$$
\Sigma_{2}=\left\{W_{2} \leq \frac{1}{2} L^{2}\right\}=\left\{\left|\eta_{1}\right| \leq L\right\}
$$

is positively invariant. If $\eta_{1}\left(t_{0}\right)$ is outside of $\Sigma_{2}$, then from (31)

$$
W_{2}\left(\eta_{1}(t)\right) \leq W_{2}\left(\eta_{1}\left(t_{0}\right)\right) \exp \left(-\lambda \frac{2 k \varepsilon_{f}^{n}}{\varepsilon_{1}}\left(t-t_{0}\right)\right)
$$

From the scaling equation (11), it can be seen that whenever $x\left(t_{0}\right)$ and $\hat{x}\left(t_{0}\right)$ are bounded, there exists a constant $k_{w_{2}}>0$, independent of $\varepsilon_{1}$ and $\varepsilon_{2}$, such that $W_{2}\left(\eta_{1}\left(t_{0}\right)\right) \leq k_{w_{2}}^{2}$. From (32) and (33), it can be seen that $\eta_{1}$ reaches the set $\Sigma_{2}$ within the time interval $\left[t_{0}, t_{0}+T_{2}\left(\varepsilon_{1}\right)\right]$, where

$$
T_{2}\left(\varepsilon_{1}\right)=\frac{\varepsilon_{1}}{\lambda k \varepsilon_{f}^{n}} \ln \left(\frac{k_{w_{2}}}{\sqrt{\frac{1}{2}} L}\right) \rightarrow 0 \text { as } \varepsilon_{1} \rightarrow 0
$$

At this point, $\eta_{1}$ is inside the strip (26) and cannot leave the strip for all future time. Inside the strip, the parameter $\varepsilon_{2}$ is driving the dynamics of the high-gain observer, not $\varepsilon_{1}$. Therefore, it is appropriate to alter the scaling equation (11) to obtain

$$
\xi=D\left(\varepsilon_{2}\right)[x-\hat{x}]
$$

where $D\left(\varepsilon_{2}\right)=\operatorname{diag}\left[1, \varepsilon_{2}, \cdots, \varepsilon_{2}^{n-1}\right]$. Then, the error dynamics become

$$
\varepsilon_{2} \dot{\xi}=A_{0} \xi+\varepsilon_{2}^{n} B \delta+B_{0} v
$$

which is valid for trajectories inside the strip. Take the Lyapunov function candidate as $W_{3}=\xi^{T} S \xi$, where $S$ is the positive definite symmetric solution to the Lyapunov equation $S A_{0}+A_{0}^{T} S=-I$. Then, it can be shown that

$$
\dot{W}_{3} \leq-\frac{1}{2 \varepsilon_{2}\|S\|} W_{3}, \forall W_{3} \geq\left(\sigma_{1} \varepsilon_{2}^{n}+\sigma_{2} \mu\right)^{2}
$$

where $\sigma_{1}=4\|S B\| L_{\delta} \sqrt{\|S\|}$ and $\sigma_{2}=4\left\|S B_{0}\right\| \sqrt{\|S\|}$. Therefore, the set

$$
\Sigma_{3}=\left\{W_{3} \leq\left(\sigma_{1} \varepsilon_{2}^{n}+\sigma_{2} \mu\right)^{2}\right\}
$$

is positively invariant. For $\xi\left(t_{0}\right)$ outside $\Sigma_{3}$, it can be seen from (37) that

$$
W_{3}(\xi(t)) \leq W_{3}\left(\xi\left(t_{0}\right)\right) \exp \left(\frac{-\left(t-t_{0}\right)}{2 \varepsilon_{2}\|S\|}\right)
$$

From the scaling equation in (35), for bounded $x\left(t_{0}\right), \hat{x}\left(t_{0}\right)$, and $\varepsilon_{2}$, there exists a constant $k_{w 3}>0$, independent of $\varepsilon_{2}$, such that $W_{3}\left(\xi\left(t_{0}\right)\right) \leq k_{w 3}^{2}$. It follows from (38) and (39) that $\xi$ reaches the set $\Sigma_{3}$ within the time interval $\left[t_{0}, t_{0}+\right.$ $\left.T_{3}\left(\varepsilon_{2}\right)\right]$, where

$$
T_{3}\left(\varepsilon_{2}\right)=4 \varepsilon_{2}\|S\| \ln \left(\frac{k_{w 3}}{\sigma_{1} \varepsilon_{2}^{n}}\right) \rightarrow 0 \text { as } \varepsilon_{2} \rightarrow 0
$$

Therefore, all of the trajectories are traveling towards the set $\Omega=\left\{\Sigma_{2} \cap \Sigma_{3}\right\}$ that is positively invariant. Moreover, the set $\Omega$ is reached within the time interval

$$
T\left(\varepsilon_{1}, \varepsilon_{2}\right)=T_{1}\left(\varepsilon_{1}\right)+T_{2}\left(\varepsilon_{1}\right)+T_{3}\left(\varepsilon_{2}\right) \rightarrow 0 \text { as } \varepsilon_{2} \rightarrow 0
$$

where the times are defined in (25), (34), and (40). Recall that $\varepsilon_{1}<\varepsilon_{2}$, meaning that decreasing $\varepsilon_{2}$ will eventually correspond to a decrease in $\varepsilon_{1}$. Hence, only the reduction of $\varepsilon_{2}$ is explicitly listed in (41).

\section{Simulation}

Consider the pendulum equation given by

$$
\begin{aligned}
\dot{x}_{1} & =x_{2} \\
\dot{x}_{2} & =\phi(x, u)=-10 \sin x_{1}-x_{2}+10 u \\
y & =x_{1}+v
\end{aligned}
$$

where $x_{1}$ is the angular position and $x_{2}$ the angular velocity subtended by the rod and the vertical axis through the pivot point. The goal is to have $\left(x_{1}, x_{2}\right)$ track the reference signals $(r, \dot{r})$, where $r$ is a step input starting at 0 and ending at $\pi / 2$ radians, passed through a second-order filter with unit gain and time constant 0.25 seconds. The motor torque is viewed as the control source and is denoted by $u$. The controller expression is $u=0.1\left(10 \sin y+\hat{x}_{2}+\ddot{r}-400(y-r)-20\left(\hat{x}_{2}-\dot{r}\right)\right)$, derived with standard feedback linearization techniques. The estimate $\hat{x}_{2}$ is saturated outside $[-3,3]$. The saturation values are chosen such that the saturation is never active when the system is under state feedback control. The nominal value for $\phi$ used in the observer is $\phi_{0}(\hat{x}, u)=0$. The gains for the observers are chosen as $\varepsilon_{1}=0.001$ and $\varepsilon_{2}=0.01$. The remaining parameters are chosen as $\alpha_{1}=11$ and $\alpha_{2}=10$. The initial conditions are set at $x_{1}(0)=x_{2}(0)=\hat{x}_{2}(0)=0$, and $\hat{x}_{1}(0)=\pi$ to match values consistent with the physical system. The initial conditions are deliberately chosen to be unequal to ensure peaking in the transient response of the system, lending itself to a more realistic scenario. The measurement noise $v$ is generated using the Simulink block "Uniform Random Number", where the magnitude is limited to $[-0.0016,0.0016]$ and the sampling time is set at 0.0008 seconds. The noise magnitude choice is based on a $1000 \mathrm{c} / \mathrm{r}$ encoder. The value of $d_{1}$ is 0.003 , while $d_{2}$ is set to 0.02 .

Fig. 2 shows the transient response of the error $\left(x_{2}-\right.$ $\hat{x}_{2}$ ). As Fig. 2(a) demonstrates, the observer with the three piece nonlinearity captures the transient performance of the linear gain observer with the parameter $\varepsilon_{1}$. Although the settling time for the observer with the two piece nonlinearity is smaller than that of the linear gain observer with the parameter $\varepsilon_{2}$, the behavior of the linear gain observer with the parameter $\varepsilon_{1}$ is not recovered. Therefore, the three piece nonlinear gain is an improvement from the previous nonlinear again approach. 

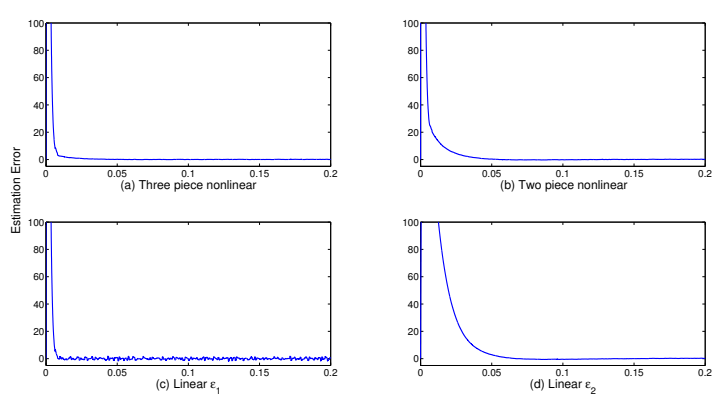

Fig. 2. Transient response of the error $x_{2}-\hat{x}_{2}$ vs. time

In Fig. 3, the steady-state behavior of the estimation error is practically identical in three out of the four observers. As expected, the linear gain observer utilizing $\varepsilon_{1}$ has a larger steady-state error due to the affects of measurement noise.

Fig. 4 shows the tracking error $\left(x_{2}-\dot{r}\right)$ during the transient response of the observer dynamics. As shown in Fig. 4, the transient response resulting from the system utilizing the three piece nonlinear-gain observer is faster than the two piece nonlinear-gain observer, but slower than the linear gain observer with $\varepsilon_{1}$. In Fig. 4(d), the linear gain observer with $\varepsilon_{2}$ yields the largest settling time.

The steady-state response of the tracking errors shown in Fig. 5 reveal that the nonlinear-gain observers are able to capture the steady-state response achieved in the linear gain observer with parameter $\varepsilon_{2}$.

Overall, the newly introduced piecewise nonlinear-gain observer is able to capture the desired system performance from the two linear gain observers.
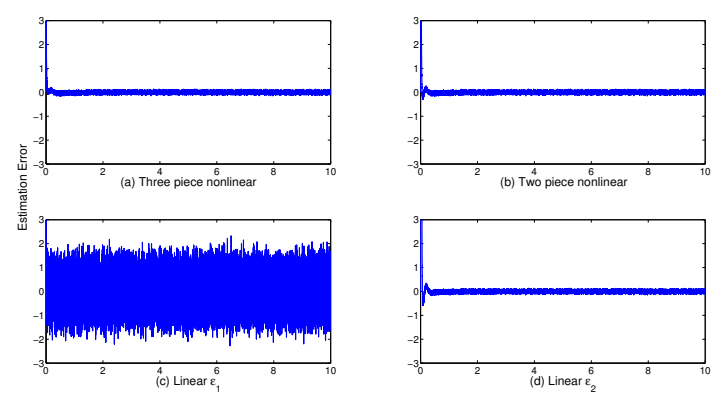

Fig. 3. Steady-state response of the error $x_{2}-\hat{x}_{2}$ vs. time

\section{Conclusions}

The intention of this work was to scrutinize the challenging issues associated with maintaining acceptable system performance in the presence of measurement noise. When high-gain observers are utilized in the presence of measurement noise, there exists a trade-off between fast state reconstruction and a reasonable state estimation error. In particular, we focused on the effect measurement noise has on the closed-loop system and high-gain observer performance, while utilizing the structure of the observer to reduce the undesirable affects of noise. The new high-gain
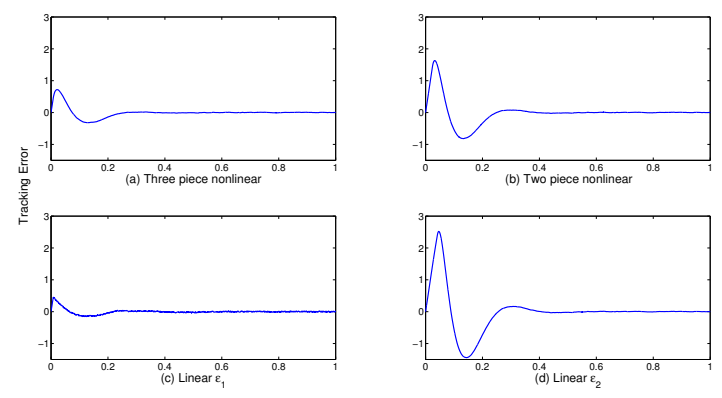

Fig. 4. Transient response of the tracking error $x_{2}-\dot{r}$ vs. time
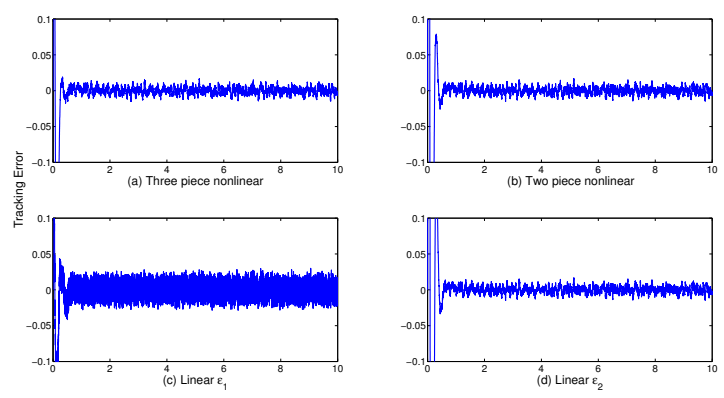

Fig. 5. Steady-state response of the tracking error $x_{2}-\dot{r}$ vs. time

observer structure with the three section piecewise linear gain surpassed the performance of all observers investigated in this paper. The nonlinearity was chosen to have a higher observer gain during the transient period and a lower gain afterwards, thus overcoming the trade-off between fast state reconstruction and measurement noise attenuation.

\section{REFERENCES}

[1] F. Esfandiari and H. Khalil, "Output feedback stabilization of fully linearizable systems," Int. J. Contr., vol. 56, pp. 1007-1037, 1992.

[2] J. Gauthier, H. Hammouri, and S. Othman, "A simple observer for nonlinear systems application to bioreactors," IEEE Trans. Automat. Contr., vol. 37, no. 6, pp. 875-880, 1992.

[3] H. Khalil, "High-gain observers in nonlinear feedback control," in International Conf. on Contr., Automat. and Syst., Seoul, Korea, 2008.

[4] H. Kwakernaak and R. Sivan, Linear Optimal Control Systems. New York: Wiley-Interscience, 1972.

[5] D. Mayne, R. Grainger, and C. Goodwin, "Nonlinear filters for linear signal models," IEE Proc. Control Theory Appl., vol. 144, pp. 281286, 1997.

[6] A. Tilli and M. Montanari, "A low-noise estimator of angular speed and acceleration from shaft encoder measurements," Journal Automatika, vol. 42, pp. 169-176, 2001.

[7] L. Vasiljevic and H. Khalil, "Error bounds in differentiation of noisy signals by high-gain observers," SCL, vol. 57, pp. 856-862, 2008.

[8] J. Ahrens and H. Khalil, "High-gain observers in the presence of measurement noise: A switched-gain approach," Automatica, vol. 45, pp. 936-943, 2009.

[9] A. Ball and H. Khalil, "High-gain observers in the presence of measurement noise: A nonlinear gain approach," in Proc. IEEE Conf. on Decision and Control, Cancun, Mexico, 2008, pp. 2288-2293.

[10] P. Kokotovic, H. K. Khalil, and J. O'Reilly, Singular Perturbation Methods in Control Analysis and Design. Philadelphi, PA: SIAM, 1999.

[11] Y. Lin, E. Sontag, and Y. Wang, "A smooth converse lyapunov theorem for robust stability," SIAM J. Contr. Optim., vol. 34, pp. 124-160, 1996. 